\title{
Training and Application of Student Standardized Patients for Dental Education
}

\author{
Xu Zheng ${ }^{1,2, \dagger}$, Wu Xiao Cui ${ }^{1, \dagger}$, Si Hui Zhong ${ }^{1}$, Peng Yao Yan ${ }^{1}$, Xun Chen ${ }^{1}$, Diwas Sunchuri ${ }^{3}$, \\ Zhu Ling Guo, ${ }^{1, \text {, * }}$ \\ ${ }^{1}$ School of Dentistry, Hainan Medical University, Haikou, PR China \\ ${ }^{2}$ Department of Dentistry, The First Affiliated Hospital of Hainan Medical University, Haikou, PR China \\ ${ }^{3}$ School of International Education, Hainan Medical University, Haikou, PR China
}

Email address:

604569033@qq.com (Zhu Ling Guo)

${ }^{*}$ Corresponding author

$\uparrow \mathrm{Xu}$ Zheng and $\mathrm{Wu}$ Xiao Cui are co-first authors.

\section{To cite this article:}

Xu Zheng, Wu Xiao Cui, Si Hui Zhong, Peng Yao Yan, Xun Chen, Diwas Sunchuri, Zhu Ling Guo. Training and Application of Standardized Patients for Stomatology Students. International Journal of Vocational Education and Training Research. Vol. 7, No. 1, 2021 , pp. 30-34. doi: $10.11648 /$ j.ijvetr.20210701.15

Received: November 22, 2020; Accepted: December 15, 2020; Published: May 27, 2021

\begin{abstract}
Background Stomatology is a discipline closely combined with theory and practice, and pays attention to students' operation abilities and clinical skills. Current dental education is encountering the situation of more theory than practice. The evaluation and application of standardized patients (SP) are gradually increasing in medical education. Objective This paper intends to describe the advantages of student standardized patients (SSP), how to construct and apply SSP for dental education. Method Recruit SP of stomatology students, and select appropriate cases based on the actual cases and common dental diseases SSP training is conducted by experienced clinical teachers. SSP is trained to describe the disease in patient's language and give a reasonable response to enquiry and examination. Result Qualified SSP can be applied in inquiry, physical examination, teaching and evaluation of common dental diseases. SSP not only provide students with repeated practice, but also enable students to understand theoretical knowledge and cultivate clinical thinking more effectively in real clinical reception. This model of SSP application could stimulate learning enthusiasm of on-campus dental student, improve student clinical skills and thinking abilities, as well as reduce education expenses. Conclusion Using SSP is beneficial to help students construct clinical thinking, learn communication skills and build a harmonious doctor-patient relationship.
\end{abstract}

Keywords: Standardized Patient, Student Standardized Patient, Stomatology, Training, Application

\section{Background}

Stomatology is a discipline closely combined with theory and practice, and pays attention to students' operation abilities and clinical skills. But in the present education, the key teaching points focus mostly on the teaching of theoretical knowledge and practice in the laboratory using isolated teeth and head simulation models. Training for clinical inquiry relatively involves the diagnosis of the disease and the techniques on how to communicate with patients and their families [1]. With the continuous enhancement of patients' awareness of safeguarding their rights, the phenomenon of asking patients to do "props" for physical examination and treatment through interns and trainees like in the past is gradually rejected by patients. The situation of more theories than practices in somatology teaching is gradually increasing [2]. Based on this social background, the appearance of standardized patients has undoubtedly brought new impetus to the development of medicine. The training and application of standardized patients for stomatology students is undoubtedly an opportunity to improve their clinical ability.

Standardized Patients (SP), also known as Simulated Patient, refers to normal people or patients who can accurately describe the patients' history and accurately present the patients' symptoms, signs and other requirements after systematic training. SP is a special group that is trained, 
accepted and issued with certificates by specialized people. Training and using of these groups requires high cost of money and time, while the training of students as standardized patients can reduce the corresponding investments. Student standardized patients (SSP) refers to medical students who can accurately present patients' actual clinical problems, and can act as teaching mentors and evaluators with triple functions after standardized and systematized training [3].

Stomatology students generally have only a preliminary understanding of their major at school. Through training, students have a deeper understanding of stomatology. SSP also has the characteristics of the faster acceptance and the shorter training time [4]. The following part will describe the advantages of standardized patients of stomatology students, how to construct standardized patients of stomatology students, and the application and prospect of standardized patients of stomatology students.

\section{Advantages}

(1) Students of stomatology in school have a partial understanding of professional knowledge. SSP resources are selected from students majoring in stomatology, and through SSP role playing and repeated practice, various professional knowledge can be improved more effectively [5]. Stomatology students who have not been exposed to clinical operation can grow interest in clinical practice and enlighten professional learning later. Students are able to integrate knowledge and skills in a clinical setting, linking diseases and laying a solid foundation for systematic learning [6].

(2) Stomatology students have some understanding of oral diseases, which can reduce the difficulty of training, reduce the expenditure of training expenses.

(3) SSP is a complex identity composed of multiple roles, which can help students determine the targets, cultivate higher social consciousness, and promote their own and professional growth [7].

(4) SP mostly participated in the form of social volunteers or part-timers, and the personnel constitute is unstable, while the students as standardized patient members are stable and can be used for a long time through training.

(5) Each SSP playing the role of oral disease may adapt to the new script as their oral condition changes and can be further utilized to achieve the maximum possible utilization of each SSP.

\section{Construction}

\subsection{Recruitment Standards}

After posting the recruitment information, let the prospective stomatology students sign up. Choose students who have a strong interest in performing among the voluntary applicants; Learning enthusiasm, high comprehensive quality;
Master certain oral knowledge; Good comprehension, expression and memory skills; Students who are in good health and free from infectious diseases.

\subsection{Case Selection}

The preparation of the case should select common diseases in stomatology, such as pericoronitis, primary trigeminal neuralgia, dental caries, recurrent aphthous ulcer, acute pulpitis, dislocation of temporomandibular joint, chronic periapical periodontitis, dental congestion, etc. Choose the right case and script the play. Compile information about the patient's education, personality, occupation, etc. The script should include the simulation of the scene, medical history, diagnosis, examination, treatment, follow-up visit, improvement, etc. And should also cooperate with props and makeup techniques when necessary [8].

\subsection{Training Implementation}

SSP training is conducted by experienced clinical teachers. After selecting the SSP and the corresponding script, SSP should be helped to master the knowledge related to the disease, familiarize itself with the characteristics of the disease and patient's reaction. The SSP is trained to describe the disease in patient's language, which should be simple and easy to understand. In the performance of the script, there can be accompanied by actions, but it is not allowed to mislead the students with exaggerated actions, nor can it be used to induce the students with implied performances [9]. Avoid using medical terminology and jargon such as "46" or "left mandibular first molar" when describing the tooth position. The SSP should be trained to give a reasonable response to the examination. For example, when performing acute pulpitis, the SSP should be trained to show a painful face, tighten the eyebrows, and make a painful appearance. It can be accompanied by language and actions, such as shouting for pain, and show a significantly relieved expression of pain after receiving open pulp treatment. The formal use of SSP repeated simulation training, helps to find out the shortcomings and to correct, and strive to achieve the best.

\section{Application}

\subsection{Inquiry}

Inquiry is the process of obtaining necessary information by systematically questioning patients or relevant personnel. Through inquiry, doctors can obtain the occurrence, development process of the disease, previous diagnosis and treatment process, allergy history, family history and other important information, so as to contribute to the further diagnosis and treatment of the present condition. The acquisition of important information is particularly necessary in the formulation of clinical treatment plans. For example, when a patient needs to have a full metal crown of the posterior tooth repaired, it is necessary to consider whether the patient has had other restorations before. Different situations will have a great impact on the choice of the plan. The two 
upper and lower teeth in the same position are repaired with different metals, which can produce a small electrical current that can cause chewing pain. The information can be obtained through inquiry, so as to avoid some unnecessary defective prosthesis made in clinical practice. Inquiry is the basis for the diagnosis and treatment of diseases, and is a particularly important part of medical education. Students can improve their inquiry skills by inquiring with the SSP to avoid blind consultation after entering the clinic [10]. SSP can be used for students to practice face to face repeatedly, which can make students pay attention to clinical communication methods and skills and improve students' confidence [11], so that students will not feel embarrassed when they come into clinical contact with real patients. It doesn't make patients tired either. At the same time, the application of SSP can enhance medical students' humanistic care for patients in daily life, cultivate the concept of caring for the wound, enhance their sense of service and sense of responsibility, and make medical students and doctors pay more attention to communication with patients. Communication skills can be trained through SSP [12], which is conducive to the construction of a good doctor-patient relationship and promote medical ethics education [13].

\subsection{Physical Examination}

Physical examination plays an irreplaceable role in stomatology. Lesions can be found by examining the skin, face, mouth, tongue and other parts of the patient, and changes in color, shape and quality of the lesion can be found. SSP vividly shows the symptoms in the selected cases, which can make the content concrete and visualized. Medical students are exposed to live cases and "patients", instead of just descriptions and two-dimensional pictures in books. After the physical examination of SSP, students will have a more virtual and concrete understanding of the disease. Some of the knowledge may be difficult to understand through simple text description, but medical students will be deeply impressed when they conduct physical examination on patients themselves which can improve their mastery of theoretical knowledge [14]. This not only enables them to pay more attention to the learning of basic knowledge and the standardization of clinical operation, but also fully combines theory with practice. It can also cultivate medical students' ability to deal with emergencies, improve their comprehensive ability, and lay the foundation for them to become an excellent doctor.

\subsection{Teaching}

SP teaching method was originally applied in medical education to teach medical students learning and communication skills [15]. The application of SSP in regular classroom makes the classroom no longer a simple model of teachers' output of knowledge. It makes the classroom interactive and responsive with vivid and diversified contents. It becomes a new type of classroom. The change of classroom improves the interest and enthusiasm of medical students in learning which is conducive to the lasting mastery of knowledge [16]. Vivid SSP can promote the abilities of stomatology students to learn independently, so as to discover, analyze and solve problems independently, and improve their self-study and problem-solving abilities. Experiments show that standardized patients can improve students' communication skills more effectively than traditional teaching [17]. Not only can SSP be applied to classroom teaching of students, but also lectures containing SSP can be designed to increase the public's awareness of some phenomena or diseases [18]. The practical teaching of SSP for stomatology students in school can make them contact with the clinical environment in advance, shorten the adaptation period after internship significantly, make easier for students to adapt to the clinical environment than other students, and play a certain role in guiding students to participate in a series of examinations and competitions [19].

\subsection{Assessment and Competition}

An SSP can act as a patient, evaluator, and teaching mentor. The development of SSP further promotes the fairness of examinations and competitions. As the evaluator, SSP has the most direct contact with students participating in examinations and competitions and has the most intuitive feelings about the whole examination and competition. It can accurately grasp students' communication, clinical skills and operation abilities, so as to make the examination more fair and just. Objective structured clinical examination (OSCE) is a kind of assessment method that uses SP to objectively evaluate candidates' clinical ability, and this method is also applicable to the evaluation of oral clinical teaching effect [20]. The introduction of SP in the objective structured clinical examination not only requires the evaluation of communication and professionalism, but also requires the evaluation and scoring of the examiner [21]. In clinical skills training, SSP communicates with students from the perspective of patients, which can help students improve humanistic care for patients from psychological, social, cultural and other aspects, and is conducive to the cultivation of patient-centered thinking [22]. SP's feedback should become a key tool [23]. The teacher doesn't give guidance to the students when they take the examination. After the examination, the teacher can summarize the students' performance based on the feedback from the SSP [24]. Students' self-evaluation is often different from SSP's feedback, which may be due to the difference in the degree of respect for patients [25]. Through the improvement of SSP's feedback, a great improvement can be achieved [26].

\section{Discussion}

SP is widely developed in foreign countries and is still in the exploratory stage in China. The first batch of SP in China was cultivated by West China Medical College in 1993 [27]. The proportion of SP used in health education and survey is also increasing gradually [28]. Many medical colleges and universities have trained SP and applied it in teaching and assessment, achieving good results. In terms of the overall 
trend, research on SSP is on the rise, especially in recent years, domestic attention to SSP training has been increasing [29]. Since standardized patients in China cannot be professionalized all the time, their large-scale use is limited. The training and use of SSP solves the embarrassing situation of lack of teaching resources, and forms a more systematic training structure that can be repeatedly used in teaching, assessment and competition.

At present, there are still some inevitable difficulties in SSP training. For example, the practice time is limited, and students of stomatology will be recruited after graduation. Some signs are hard to imitate. Repeated performance will make the SSP tired and so on. The duration can be increased by recruiting lower grade stomatology students. To increase the interest and enthusiasm of SSP, certain subsidy and certificate can be provided to SSP with excellent performance.

In clinical practice, with the transformation of modern medical model, it is more and more advocated to build a good doctor-patient relationship. To achieve this, excellent professional skills and good medical environments should be provided for patients. Effective communication with patients can undoubtedly promote the construction of a good doctor-patient relationship [30]. Patients' understanding of the disease will affect their treatment compliance and prognosis, so communication is very essential for doctors, and SP provides an opportunity to carefully study communication skills [31]. The standardized use of patients by stomatology students can not only help medical students learn to think in others' shoes and increase their awareness of caring for patients but also improve the ability to analyze and solve problems, improve the ability to communicate with patients and gain experience [32]. It also helps to improve the ability to communicate with team members and enhance teamwork [33]; arouse the SSP's professional ethics and feelings, improve the post competency which is conducive to a comprehensive grasp of basic knowledge [34]. The use of standardized patients for stomatology students can not only exercise and assess students' clinical skills, but also increase the consideration of ethical issues, such as tooth extraction for hepatitis $b$ viral-positive patients [35]. This model of using stomatology students to SSP not only reduce expenses, but also stimulate SSP learning enthusiasm, improve clinical skills and thinking abilities, to achieve a win-win situation, and cultivate high-quality oral talents.

\section{Conclusion}

Based on the importance of stomatology practice and clinical teaching, the evaluation and application of standardized patients are gradually increasing. Stomatology student standardized patients have its unique advantages. The training particularly involves important events including recruitment, clinical case selection, and training implementation. It can be applied in the inquiry, physical examination, teaching and evaluation of common dental diseases, which is conducive to cultivate high-quality oral talents.

\section{Fund}

This research was funded by Education Department of Hainan Province (project number: Hnjg2021-60), Course Construction Project of Hainan Medical University (HYJW202117), Educational Scientific Research of Hainan Medical University (HYYB202014), Marxism Theory Discipline Research Project of Hainan Medical University (QMYKYLX201807), Research Project of Hainan Provincial Humanities Medical Research Base (QRYZH201811 (YB)), Innovative Entrepreneurial Training Program of Hainan Medical University (X201911810027).

\section{References}

[1] Xiao-Lin LV, Liang XU, Bu-Ling WU, Wen-An XU. Application of Standardized Patients in Stomatology Teaching [J]. Southern Medical Education, 2019, 30 (02): 42-44.

[2] Hong DONG, Ming-Sheng LONG, Cai-Yun LIU, Xiao-Wei QU, Xiang DONG, Yang YANG. Application of Standard Patients in China Clinical Teaching of Stomatology and the Analysis [J]. Journal of Hanjiang Normal University, 2014, 34 (04): 132-135.

[3] Bi-Yuan QIN, Juan LIAO, Yi-Ning WANG, Cong-Min JING. Application of Student Standard Patient in the Intern Pre-job Training [J]. China Continuing Medical Education, 2020, 12 (18): 61-63.

[4] Park Yoon Soo PhD, Hyderi Abbas MD MPH, Heine Nancy MEd RN ANP, et al. Validity Evidence and Scoring Guidelines for Standardized Patient Encounters and Patient Notes From a Multisite Study of Clinical Performance Examinations in Seven Medical Schools. 2017, 92 (11S): S12-S20.

[5] Lei WANG, Meng CAO, Lian-Cheng WANG, Jiao-Jiao HE, Shan-Min FU. Application of Students as Standardized Patients in the Teaching of Orthodontics [J]. Basic Medical Education, 2020, 22 (04): 274-277.

[6] Jackson Jennifer M, Strowd Lindsay C, Peters Timothy R. The Simulated Virology Clinic: A Standardized Patient Exercise for Preclinical Medical Students Supporting Basic and Clinical Science Integration [J]. Mededportal: The Journal of Teaching and Learning Resources, 2020, 16.

[7] Samantha A. Starr, Margaret M. Plack, Karen L. Lewis, Benjamin Blatt. How Do Standardized Patients Form Their Complex Identities? The Impact of Interactions With Medical Students [J]. Simulation in Healthcare: The Journal of the Society for Simulation in Healthcare, 2020.

[8] Xi DENG, Jia-Mei TAN, Shu-Yuan PAN. Application of "Standardized Patient" and "Standardized Family" Teaching Model in Training Medical Students' Doctor-patient Communication Skills [J]. Journal of Shenyang Medical College, 2019, 21 (05): 486-488.

[9] Sheng-Mao MA, Hua ZHANG, Hai-Bo YANG, Yu-Rong GE, Jing ZHOU, Tao YANG. Standardized Patient Training and Its Application in Clinical Teaching and Assessment [J]. Education Teaching Forum, 2019 (02): 73-75. 
[10] Deborah Byrne. Evaluating Cultural Competence in Undergraduate Nursing Students using Standardized Patients [J]. Teaching and Learning in Nursing, 2020, 15 (1).

[11] Hattie H. Wright, Judi Cameron, Tania Wiesmayr-Freeman, Libby Swanepoel. Perceived Benefits of a Standardized Patient Simulation in Pre-Placement Dietetic Students [J]. Education Sciences, 2020, 10 (7).

[12] Amy Whittle, Sarah Bourne, Summer Segal, Janet R. Serwint. Teaching Death Disclosure: A Mixed-Method Comparison of Resident Self-Assessment and Standardized Patient Assessment [J]. Academic Pediatrics, 2020, 20 (5).

[13] Mahendran Rathi, Lim Haikel Muhammad Asyraf, Kua Ee Heok. Medical Students' Experiences in Learning the Mental State Examination with Standardized Patients [J]. Asia-Pacific Psychiatry: Official Journal of the Pacific Rim College of Psychiatrists, 2019.

[14] Qian REN, Min LIU, Ya-Li YAO, Rui JI. The Application and Valuation of Student Standardized Patients in Diagnostics Teaching [J]. China Continuing Medical Education, 2020, 12 (14): 58-61.

[15] Megan M. Keiser, Carman Turkelson. Using Students as Standardized Patients: Development, Implementation, and Evaluation of a Standardized Patient Training Program [J]. Clinical Simulation in Nursing, 2017, 13 (7).

[16] Javidi Sarafan Maryam, Tafazoli Mahin, Khadivzadeh Talat, Mazloum Seyed Reza. Comparing the Effect of Standardized Patient-based Education and Feedback Lecture on Midwives' Knowledge and Practice in Counseling Screening for Fetal Malformations [J]. Journal of Education and Health Promotion, 2020, 9 .

[17] Geoffroy Pierre A, Delyon Julie, Strullu Marion, Dinh Alexy Tran, Duboc Henri, Zafrani Lara, Etienne Isabelle, Lejoyeux Michel, Ceccaldi Pierre-François, Plaisance Patrick, Peyre Hugo. Standardized Patients or Conventional Lecture for Teaching Communication Skills to Undergraduate Medical Students: A Randomized Controlled Study [J]. Psychiatry Investigation, 2020, 17 (4).

[18] Ross Mary Ellen Trail, Bryan Jennifer L, Thomas Katharine L, Asghar-Ali Ali Abbas, Pickens Sabrina L. Elder Abuse Education Using Standardized Patient Simulation in an Undergraduate Nursing Program [J]. The Journal of Nursing Education, 2020, 59 (6).

[19] Guang-Lun LI, Li-Qiu LIU, Zuo-Yuan CHEN, Jun WU, Rui-Xia MA. Training of Junior Medical Students as Standardized Patients and Its Application in Simulation Teaching [J]. Journal of Qingdao University (Medical Sciences), 2020, 56 (04): 489-491.

[20] Xing-Yuan SUN, Jia JI, Xiao-Hua LI, Li LIU, Yuan-Zhi XU. Application of Mini-CEX and OSCE to Improve the Quality of Stomatology Clinical Practice [J]. China Continuing Medical Education, 2019, 11 (16): 14-17.

[21] Roy Marguerite, Wojcik Josée, Bartman Ilona, Smee Sydney. Augmenting Physician Examiner Scoring in Objective Structured Clinical Examinations: Including the Standardized Patient Perspective [J]. Advances in Health Sciences Education: Theory and Practice, 2020.

[22] Defenbaugh Nicole, Chikotas Noreen E. The outcome of interprofessional education: Integrating Communication Studies into a Standardized Patient Experience for Advanced
Practice Nursing Students [J]. Nurse Education in Practice, 2016, 16 (1).

[23] Schwartz Alan, Peskin Steven, Spiro Alan, Weiner Saul J. Direct Observation of Depression Screening: Identifying Diagnostic Error and Improving Accuracy Through Unannounced Standardized Patients [J]. Diagnosis (Berlin, Germany), 2020.

[24] Zhen NING, Xiao-Yan CHEN. Application of Student Standardized Patients in Medical Nursing Practice Assessment [J]. Academic Research of JXUT, 2019, 14 (03): 21-24.

[25] Jihye Yu, Sukyung Lee, Miran Kim, Janghoon Lee, Inwhee Park. Changes in Medical Students' Self-Assessments of Clinical Communication Skills after Clinical Practice and Standardized Patient Feedback [J]. Academic Psychiatry, 2020, 44 (3).

[26] Schwartz Alan, Peskin Steven, Spiro Alan, Weiner Saul J. Impact of Unannounced Standardized Patient Audit and Feedback on Care, Documentation, and Costs: an Experiment and Claims Analysis [J]. Journal of General Internal Medicine, 2020 .

[27] Stillman P L, Wang Y, Ouyang Q, et al. Teaching and Assessing Clinical Skills: A Competency-based Programme in China [J]. Medical Education, 2010, 31 (1): 33-40.

[28] Davies Ellen L, Pelentsov Lemuel J, Montagu Adam, Gordon Andrea L, Hooper Kenneth J, Esterman Adrian J. "Who Am I and Why Am I Here?" A Scoping Review Exploring the Templates and Protocols That Direct Actors in Their Roles as Simulated (Standardized) Patients [J]. Simulation in healthcare: journal of the Society for Simulation in Healthcare, 2020.

[29] Xin-Tong LIU, Hao-Jing TANG, Yao XIAO. Current situation of Student Standardized Patients training in China [J]. China Medical Education Technology, 2020, 34 (02): 184-186+190.

[30] Chuan WEN, Jie XIONG, Cheng WANG, et al. SimBaby Plus Standardized Patient Teaching Model in the Teaching of Cases of Acute and Severe Bronchopneumonia in Infancy [J]. 2017, 33 (9): 630-634.

[31] Jeffrey A. Wilhite, Khemraj Hardowar, Harriet Fisher, Barbara Porter, Andrew B. Wallach, Lisa Altshuler, Kathleen Hanley, Sondra R. Zabar, Colleen C. Gillespie. Clinical Problem Solving and Social Determinants of Health: A Descriptive Study Using Unannounced Standardized Patients to Directly Observe How Resident Physicians Respond to Social Determinants of Health [J]. Diagnosis, 2020, 7 (3).

[32] Elif Ok, Fatma Yasemin Kutlu, Elif Ates. The Effect of Standardized Patient Simulation Prior to Mental Health Rotation on Nursing Students' Anxiety and Communication Skills [J]. Issues in Mental Health Nursing, 2020, 41 (3).

[33] Mariya Tankimovich, Jennifer Swails, Marcelle Hamburger. Nurse Practitioner and Medical Students' Perceptions of Teamwork Before and After a Standardized Patient Pilot Simulation [J]. Nursing Education Perspectives, 2020, 41 (3).

[34] Hong-Mei ZHANG, Jun-Yi NIU, Yu-Zhen NING, Jian-Liang QIAO. SP + CBL Teaching to Cultivate Medical Students' Humanistic Quality [J]. China Continuing Medical Education, 2020, 12 (24): 73-75.

[35] Kucukkelepce Gulhan Erkus, Dinc Leyla, Elcin Melih. Effects of Using Standardized Patients on Nursing Students' Moral Skills [J]. Nursing Ethics, 2020. 